\title{
New Open Digital Marketplaces: Giving Buy, Sell and Trust Control Back to Users
}

\author{
Vrinda Almadi, Anmol Saxena, Sarvjeet Herald* \\ Department of Computer Science and Digital Technologies, RoboGenius, Gurgaon, India \\ Email address: \\ vrinda.almadi@gmail.com (V. Almadi),anmol@robogenius.in (A. Saxena), sarvjeet@robogenius.in (S. Herald) \\ ${ }^{*}$ Corresponding author
}

\section{To cite this article:}

Vrinda Almadi, Anmol Saxena, Sarvjeet Herald. New Open Digital Marketplaces: Giving Buy, Sell and Trust Control Back to Users. American Journal of Management Science and Engineering. Vol. 5, No. 1, 2020, pp. 1-9. doi: 10.11648/j.ajmse.20200501.11

Received: December 29, 2019; Accepted: January 7, 2020; Published: January 23, 2020

\begin{abstract}
Companies such as Amazon, Flipkart, Zomato, Oyo and Uber which we refer to as Digital Marketplaces are globally emerging across different business sectors. These marketplaces connect buyers and sellers and the marketplace owners act as central trustworthy authority to provide trade assurances. However, such a trust model could be problematic. Without any alternatives, rapidly growing users of the marketplace, both buyers and sellers are forced to trust the marketplace owner to satisfy their needs. Regulating users, control over digital transactions, exercising autonomy are other issues to name (but) a few indicating that marketplace owners are transparently replacing governments. In our view, Digital Marketplaces should be democratic as the markets in the real-world and control must be given back to the users since they are the contributors in the growth of these marketplaces. This paper presents a new way of Open Digital Marketplaces that model real-world buyer and seller interactions. Proposed solution involves users of marketplace as partakers in a digital transaction for connecting buyers and seller with trade assurances by leveraging their personal connections. Trust is made localized and the supply chain becomes subjective to the user as in the real-world. Switching the role of marketplace owner with the users, eliminates the need for trusting the marketplace owner as a central authority as well as diminishes autonomy and authority of the marketplace owner over the digital transactions. Users, with whose efforts trade is made digitally successful, are rewarded with an incentive, a type of shareholding in the digital transaction, as per the electronic contracts digitally signed by participants before executing the transaction. Disputes or violations of contract are resolved by an automated system, can also be escalated to an independent body or to a local court for a decision. Feedback received from the experiment based on the implementation of the proposed solution suggested ease of doing business by small businesses as well as increase in revenue. It also creates new opportunities for users to earn revenue share in digital transactions, instead of only the marketplace owners, in the emerging gig economy.
\end{abstract}

Keywords: E-commerce, Digital Marketplaces, Trust, Social Media, Digital Democracy, Gig Economy, Management

\section{Introduction}

Digital technologies should complement physical processes to create new opportunities for the citizens. In this regard, World Wide Web, for example, transformed the way people consume information. But more recently, its creator has been reflecting on how the original idea of web has deviated to now being dysfunctional [1]. He argues that such an approach is only working well for few companies which are growing at a massive scale but at an expense of human rights and democracy. People who use these platforms are not the real beneficiaries when they should be and earning a fair share for their contributions in the growth of organizations.
This, in a truest sense if happens, would reflect a fair gig economy, where people apart from making money will also be able to exercise their rights as shareholders of the digital-enabled organizations. Contract for web is one alternative to give power back to the people as it will add transparency and build confidence among people.

Focusing on e-commerce in this paper, more specifically Digital Marketplaces, we discuss that the autonomy exercised by the marketplaces owners is creating an undemocratic digital environment. They may act biasedly to achieve greater profits, may adopt methods which would create a misleading perception of high trust in their organization among people whilst denying them rights and are gradually replacing 
governments. Societal consequences may be severe. For example, it may lead to unemployment and non-competition. Many small businesses are not able to compete with the design, strategy, pricing and policies of the marketplace owners and are running out of business [2].

Building upon the real-world approaches, the objective of this paper is to propose an alternative that may be built upon current Digital Marketplace solution which is open and not controlled by marketplace owner. It is achieved by adding a new trust methodology based on personal connections which includes shareholding in the digital buy and sell transactions to a wider participants and independent resolution of disputes, all happening in an undemocratic manner. We envisage that such an approach has potential to generate employment for many in the emerging gig economy, minimize reliance and authority of marketplace owners and give rights and power back to the people and governments.

Next section discusses the evolution of Digital Marketplaces and categorizes them based on their business models to understand issues in the Digital Marketplaces. Section 3 discusses the trust models used by marketplaces owners to develop confidence among the people and persuade them to buy and sell. Section 4 presents the proposed solution. Section 5 discusses the implementation of our solution in the real world. Finally, the last section concludes this article.

\section{Digital Marketplaces}

Notion of buy and sell between a business and a consumer changed with introduction of World Wide Web in 1990s. Instead of brick and mortar stores, websites provided convenience of shopping to a consumer from anywhere and anytime. Dell in this regard, starting in mid-90s and still successful today, is an excellent example of e-commerce which refers to situation when a company sells its own products digitally on their website. Amazon, for example, started on the same lines at the same time as Dell. It started with selling music, videos and books of other companies, but later shifted to include small businesses to sell their products and services.

Similar to Amazon, Craigslist around the same time began its operations by allowing sellers to post classified advertisement and distributing it via emails to friends and in their social circle. eBay's venture created opportunities for consumers to digitally sell or re-sell to other consumers. Moving from e-commerce store containing product catalogue of their own, approaches have evolved over the years into Digital Marketplaces where owners of their websites allow multiple sellers (retailers, manufacturers, producers or even consumers) to populate product list and sell their products to consumers through a common website. Such an approach was adopted, for example, by Amazon.

As the digital devices are ubiquitous, not-in person (where users are not physically present) transactions are taking place naturally and are exponentially growing [3]. This section briefly categorizes Digital Marketplaces to highlight drawbacks in the existing approaches and demonstrate need for this work.

\subsection{Single Seller Digital Marketplaces}

Popular websites such as dell.com and apple.com fall in this category. These websites act as digital stores of the counterpart physical stores with the objective of reducing the brick and mortar costs, manpower and the logistics costs. High advertising and marketing budgets are used to inform, persuade and remind customers about the brand or its products. With strong motivation, conviction or social acceptance, buyers visit these digital stores to fulfill their wish.

Sellers or retailers of the company are not allowed to trade on these websites. However, their contact details are listed in case buyer wishes to inspect, experience or buy from a local physical store. Such websites make revenue from the success of their marketing campaigns, market hype and customer loyalty. Since the budget and end-to-end costs of single brand digital marketplaces are high, it becomes difficult for small businesses to compete. These marketplaces also do not provide perspective on the market landscape, for example, similar products, features and prices offered by the competitors in order to minimize options shown to the visitors and promptly sell.

\subsection{Classifieds Digital Marketplaces}

Popular websites such as craigslist.com, quikr.com and olx.in fall in this category. These websites allow sellers to advertise their products on the marketplace, but their owners do not govern any central authority. There are no restrictions on the number of advertisements posted by the seller, but each advertisement must consist of a single item for sale. Such marketplaces are extensively used for re-sale items by individuals and small businesses.

Since there is no option to create a catalogue of products for a seller, often duplicate entries of the same item exist in the database. As a result, database management is problematic and user interface may appear chaotic. Team of reviewers, which are gradually being replaced by Artificial Intelligence algorithms, regularly check the new advertisements posted by seller to avoid similar or identical repetitions, especially from the same seller. They also validate if the advertisements are posted within correct geographical region of seller. Each advertisement is accompanied with a lifetime (e.g., thirty days) which is mostly set by the website owner or until it is sold.

Such marketplaces mostly do not charge the seller and/or the buyer, nor it gets involved in the payment handling. It acts as mere introducer on local offerings to the parties without any obligations, especially in the case of disputes. This is because the transaction happens offline between the seller and the buyer. Since the sales are localized, buyers can conduct in-person inspection of the item and develop firsthand confidence in the seller before making the purchase. In-fact website owners recommended physical inspection before purchase.

On completion of the sale or removal of the advertisement, feedback in the form of reviews and ratings are requested from 
both the parties. These are used by the marketplace to develop confidence in the future transactions by either of the parties. Other users of the marketplace use these metrics to determine genuineness of the sellers and of buyers.

Localized approach and in-person inspection limit such marketplaces to a geographical region. They are not able to satisfy needs and wants of people across geographical regions. It is important to overcome this barrier and allow possibility of in-person or at least delegated inspections across the geographical authorities but without introducing a new central authority [4]. Similar to multinational organizations having offices and representatives at multiple regions, assigning digital representatives in the vicinity of the seller in return for a fair incentive may address limitations of such marketplaces.

\subsection{Multi Seller Digital Marketplaces}

Websites such as amazon.com, flipkart.com, taobao.com, zomato.com, oyorooms.com, bigbasket.com, ebay.com, uber.com and olacabs.com fall in this category. Sellers can populate their catalogue. Built-in search engines are used by buyers to discover the product of their choice. Recommendations in the form of search results are based on text relevancy, availability of the stock, price, shopping preferences of the buyer and purchase history of the buyer. Other factors that affect the recommendations are sales velocity of the seller, quality of images, clear product description, product storytelling and feedback. Apart from text-based search, these marketplaces are gradually introducing visual search feature which allows buyers to search catalogue for identical or similar items using photos. Wish to buy a product mostly accompanies other recommendations from the marketplace in the pursuit of upselling and cross selling products from one or more sellers.

It is important to note that the buyer wishes to buy product from the marketplace. At least the big marketing campaigns of the marketplaces create an illusion among the citizens that he/she is purchasing the product from the marketplace. In reality, sellers associated with the marketplaces across geographical locations are selling the product, not the marketplace. As a result, marketplace always receives higher visibility than the seller in digital transactions and instead of buyers developing confidence in the seller, buyer starts to develop confidence in the marketplace who is not the seller of the product and repeats a purchase but unknowingly often from another seller [5]. As a result, profits and authority of the marketplace is on rise, but seller is losing the opportunity of revenue increase in the long-term.

Another risk may arise from the Artificial Intelligence algorithms that are learning the purchase and selling behaviors of buyers and sellers on the platform. Marketplaces stock up most popular products or start to produce these products and use their authority to directly sell those products to the buyers at lower selling prices, forcing other sellers to run out of business. They might also leverage human intervention in recommendations. More recently, search engines in general are being a subject of review for tweaking their algorithms for personal gain. Such a biasness leads to loss of trust and opportunities for other sellers, especially within marketplace.

Unlike Classifieds Digital Marketplace, monetary transactions are always handled by the Multi Seller Digital Marketplaces. One reason for this is to mitigate challenges of digital transactions such as cheatings, fraud and deceptions. However, in case of a dispute, decision of the marketplace owner is final. It is supposed to be honored without buyers and sellers having any option. Consequences can be severe, for example, marketplace may block account, apply compulsory penalty or even prevent from transacting with another seller within the marketplace. Since users are forced to obey and not challenge the authority of marketplace, disputes are resolved quickly (mostly in one or two communications but) without taking into consideration that either of the party remains unsatisfied with the decision. Buyers and sellers don't have a voice, much choice in the marketplace and are not able to exercise their rights as in the real world [6].

Autonomy of marketplaces can be problematic. They can, for example, limit sellers from the money they can make in a day by looking at their sales velocity. Accounts may be closed temporarily due to policy violations which are of continuously changing nature. Suppose when a food delivery driver denies service for a digitally paid order and buyer requests for refund then marketplace may deny refund citing that the order got cancelled because buyer was unresponsive at the time of delivery. Buyer now needs to pay a cancellation charge equal to the already prepared/dispatched order amount. In case of Pay Cash on Delivery, the penalty is added to the account and user is required to pay it in addition to the next order which could be from the same seller or a different one. Buyer remains helpless if he/she wants to use the marketplace again to satisfy his/her needs. Same may apply for the sellers.

In the real world, if a buyer is not satisfied with the service of the seller, he/she may decide to never return back again to that seller as there are plenty of other sellers in the market. Sellers or their formal association will never boycott a buyer or never deny a service to the affected buyer, except in cases such as abuse. However, if users of the Digital Marketplace are not obedient, they may be denied service, even from a different seller.

Since marketplaces collect digital payments from buyers and wait for a self-determined lock-in period before releasing the money to the seller, it may decide not to return money to either of the parties based on the reviews, rating, inactivity or dispute in the transaction citing their policies. It may not have an incentive to oblige disputed parties all the time. One may decide to go to consumer courts but given the amount of the transaction in contrast to the size, authority and resources of such marketplaces, legal proceedings may not justify to the buyer, seller or both. The whole system may appear steamrolling to the buyer and/or seller because of a central authority exercised by the marketplaces [7]. As a result, current environment is implicitly undemocratic and requires review.

\subsection{Social Digital Marketplaces}

Social Media websites such as facebook.com, WhatsApp or 
WhatsApp Business and Instagram.com fall in this category. These have been widely popular among digital community to show-off their (digital or offline) purchases among their friends or social circle. This often leads to social proof for peers and persuading them to do something what others are doing for their social acceptance in their social circle but without explicitly telling them to do it. Such companies are often owned by a single parent company. Given their size, growth rate and influence, they are transparently (not explicitly visible manner) replacing governments and could pose threat if not addressed [8]. Building upon the persuasion to own something that is generated by Social Digital Marketplaces, there are three popular approaches.

First approach focuses on learning the user behaviors and interests from their interactions in the social circle and creating their virtual identities. This is often extended to masses to develop personas which are often traded with Single Seller Digital Marketplaces for targeted digital marketing and advertising [9]. People, the users of the marketplace, who are contributing to this information which is of confidential nature are largely missing on the sale of their data. In our view, anonymizing data is not a desired solution. It is similar to the practice of curation, where the original owner requires compensation or voluntarily giving up rights. But in the case of marketplaces this is not voluntary. Users are forced to give up rights in order to continue using marketplaces.

Second approach is more localized to a geographical area where people re-sell products to group of known people. This approach works similar to the Classifieds Digital Marketplace but with an added feature that the group members can further broadcast among their network of friends. Thus, making buy and sell transactions more trustworthy [10]. However, there is a little incentive to do such an activity due to lack of personal rewards or shareholding in the digital transaction.

Third approach focuses on Single Seller Digital Marketplaces actively recruiting people with large following or a significant social circle. These are referred to as influencers who contribute in the development of brands and promote their digital sales. This approach often diverts the interested people to their Single Seller Digital Marketplaces for completing the sale. Depending on popularity, influence and generated revenue, influencers are compensated by these marketplaces. Reward acts as an incentive to continue promotion and retain employment by individuals in the emerging gig economy.

It is important to learn that, in either of the cases, success of digital trading lies in understanding and leveraging personal connect with other people, their personal experiences and recommendations. Especially, when minimal number of people are involved in the transaction, it becomes more likely that the captivated interest would be converted into a sale [11]. Although such approaches start with distributed nature (where people you trust introduce you to the seller), however sales largely remain localized to a geographic region where the buyer and the seller are in close vicinity. There is a growing need to extend the localized nature of trading to a more globalized scope, whilst incorporating social phenomenon, which models the real-world nature of transactions using personal connect for developing trust among strangers.

\section{Existing Trust Models}

Several digital trust models have been proposed in the literature to develop relationships among people who are not personally known to each other ("strangers"). Many models implicitly assume trust to be transitive and build upon such an approach [12]. Even the Social phenomenon, as discussed in the previous section which models the real-world nature of transactions using personal connect, is transitive in nature when implicit chains of recommendations are formed. In this section, we discuss existing models relevant to our work to demonstrate our motivations for an improved method to enhance trust in a non-transitive manner in marketplaces without the need of a central authority to act as an arbiter during digital transactions.

\subsection{Direct Trust}

Early cryptographic techniques assumed trust to be two way. Secret was shared between Alice and Bob who knew each other personally to decrypt the message. But, let's say Alice also trusts Eve. Although Alice may trust Bob, but the same might not hold true for Bob trusting Alice. There is no guarantee that Alice will not pass on the secret, it was only an implicit assumption born out of personal connect that both Alice and Bob will behave responsibly. Therefore, trust is best suited direct and one way as in the real world. This has been evident in Single Seller Digital Marketplaces and also in Social Digital Marketplaces where people have faith in their influencer. But it can be problematic to assume that the influencer will also trust his/her followers. If this was the case, both influencer and their followers would have equal rights and shareholding in the digital transaction.

\subsection{Hierarchical Trust}

Multi Seller Digital Marketplaces follow the hierarchical trust model. They act as a central trustworthy authority to provide trade assurances. But not just for individuals, this has also been a growing concern for governments. Often chains of trust are developed and extended with central authority acting as the arbiter. This trust model represents a parent-child relationship where a single parent can have multiple children who trust their parent.

If the parent, for example, is a Multi Seller Digital Marketplace, then children are the buyers and the sellers on the marketplace. But in contrast to the real world where children have prior personal relationship with their parents, user of the digital marketplaces have no prior personal relationship with the marketplace owner and vice-versa. Contrary to the real world where parents are responsible for healthy upbringing of their children, marketplaces are focused towards commercial gains and not the well-being and rights of their users. They are transparently replacing governments who are regulators in the real world but with a different objective. 
Power switch is, therefore, a growing concern worldwide and measures are required to mitigate this risk.

Assumed power switch has raised other concerns. Over the time, marketplaces track actions of buyers and sellers and analyze it to understand their behavior. But if governments try to do the same, they receive large resistance in a democratic world. Since users have no choice or rights in the marketplace, their data is sold (although anonymized as claimed) to third parties for target-based marketing and advertising. In return, children are rewarded with the promise of better personalized services but without any monetary incentive. It would be hard to understand that in real world, a morally sound parent would be trading their children for a commercial incentive.

In the real-world, children can exercise rights over their parent's assets. But digital world does not provide that kind of a provision. Users of marketplaces have no alternatives. Hence, we press on the need for undemocratic nature of the marketplaces and giving rights back to the users. Their governments on regaining powers may choose to utilize this as an opportunity in the emerging gig economy to also provide minimum basic income to its citizens.

\subsection{Reputation Systems}

Reputation Systems are gaining popularity to overcome fraud, deception and suffering of innocent people during digital transactions and developing confidence among strangers [13]. The idea is to aggregate feedback about sellers or buyer's past behaviors and showcase these metrices to other users and allow them to contemplate whether their digital transaction with the intended buyer or seller will be satisfying.

Feedback or experience is often recorded in the form of a rating point and reviews which is used by buyers to determine credibility of the seller and thus make a purchase decision. Since marketplaces provide assurances on the digital transactions, these Reputation Systems are of a little value. People may always remain in the illusion that he/she is purchasing the product from the marketplace and hence they are protected by the marketplaces.

However, if one still persists on using Reputation Systems, reciprocity in feedbacks may distort actual behavior and lead to inefficient outcomes in marketplaces [14]. They may still be biased [15]. Buyer-seller or marketplace-seller may interact in social manner, omit negative information in reviews and may mislead others. Although Reputation Systems may be perceived as a promising solution to minimize discrimination in marketplaces, but they may not be always effective as desired. For example, in case of a motorcycle rental platform, results show that renters with an ethnic minority background receive fewer reviews than renters with ethnic majority [16]. Over the time these inequalities may grow, become incomparable and lose trust in the system.

\subsection{Non-Transitive Trust Model}

Trust* ("trust star") is a novel way of building on existing trust relationships using an electronic equivalent of real-world guarantees so as to avoid the need for transitive trust [17]. It provides a flexible way to bridge the trust gap between the strangers using localized guarantees. Each person only directly trusts the person who provides them with a guarantee. A payment model is used for motivating guarantees in the Trust* protocol. These include commission payments, as an incentive, for giving a guarantee and penalty, as a deterrent, for defaulting a guarantee and also compensating the affected parties. Concept of extending trust, using localized guarantees, enables the truster (person who is trusting a person) to act as if he trusts the trustee (person who is being trusted) directly.

Such an approach can be extended to a Web of Trust* ("web of trust star") that does not have a central authority exercising autonomy and does not rely on an unknown third party to develop trust relationships [18]. All Assurances are provided by the guarantor whom the user directly trusts. Buyers and sellers need not trust each other. Instead, they use Trust* path to develop trust which remains localized and direct. In the situations where trustee suspects truster, he/she can choose an additional route from a different direct trust relationship to provide assurances in reverse direction.

In next section, we demonstrate that an application of such a Web of Trust* would help address issues in marketplaces and make it open. Next section discusses our proposed solution.

\section{Proposed Solution}

Web of Trust* is used for introducing localized and direct trust in marketplaces. It works on personal connections similar to Social Digital Marketplaces. But in our case, personal connections provide recommendations along with guarantee. This helps to develop confidence among buyer and sellers who are strangers. There is no central controller, arbiter or a central authority exercising autonomy during the digital transactions, it is replaced by personal connections.

Democratic nature of the transactions is evident from the fact that buyers and sellers are not imposed decisions by the marketplace owner because it is no longer controller and is replaced by users. There are contracts and dynamic supply chain consisting of none, one or more persons involved with each having shareholding in a digital transaction. Disputes such as cheatings, frauds and deceptions can be resolved locally by working along with the people involved in the transaction. With users in-charge of the marketplace, governments can implement their jurisdiction, disputes can be escalated to an independent body of reviewers or at local courts in the real world. Citizens receive an opportunity to directly earn in the emerging gig economy or indirectly from their governments.

Modelling the real-world trade transactions, our proposed solution is divided into five stages. These are Registration, Discovery, Supply Chain, Contract and Delivery, and Rewards and Dispute. Users can broadcast their intention to sell or purchase and choose whom to involve for executing the transaction and resolving the disputes. 


\subsection{Registration}

In order to get associated with the platform, people can register on the platform. They may use the approach of single sign-on. However, as we are modelling the real world into a digital space and since financial transactions are of importance to the governments, we envisage that their participation may add value. A social credit system, virtual identity or social security number linked to their government's revenue system could enhance auditability, accountability and introduce good governance. The registration process can be flexible. However, efforts must be made to mitigate the redundancy in profiles by users.

\subsection{Discovery}

Discovery of a product, buyer or a seller takes place in this second stage. When no transactions have taken place, seller initiates the action. Seller creates a directory of people, whom he/she knows. These might be family connections or the existing customer base whom he/she has interacted and delivered positive experience in the real world. Existing communication methods could be used to broadcast their digital stores in the Open Digital Marketplace. This will encourage their direct connections to get associated with the seller. Since these direct connections are known to the seller personally, they trust seller based on their previous real-world experience. Moreover, income sharing option acts as a persuasion to the receiver and a motivation to join the network of seller for co-promoting sales.

People who accept the invitation of the seller are required to rate their past experience with the seller on a scale of 1 to 10 . This numerical rating forms the trust score of the seller and also how much trust truster (direct connections) has in the trustee. Our proposed solution does not recommend extending the use of this score beyond the two people. This is because and as discussed earlier, such trust scores may be of little importance to the strangers when making their purchase decisions. However, similar to the stock prices of companies which are regularly fluctuating based on the sentiments, opinions and the performances of the seller, these culmination of trust scores towards a seller from truster may become relevant in future as the indicator of the confidence of the community in the business and may be used for investing in a business venture. Such an approach may also create opportunities for government in overlapping cyber and physical world.

Existing methods could be used to circulate the catalogue of the seller among their trusters. A person has on average 338 friends on Facebook [19]. Assuming that this is a general figure of our friends in a digital social network and is a significantly small number in contrast to the entire digital population, our proposed solution will not cause network congestion. Even though small, these people which we call influencers are used in our proposed solution to promote sales unlike Social Digital Marketplaces where influencers with large fan base were especially recruited by businesses.

With this approach, sellers are no longer advertising their products similar to a classified but have started to attract the small number of people who trust his/her business and leverage them to actively engage the markets' interest in his/her catalogue. This also works well for the emerging gig economy. Trusters can earn a better livelihood for their contributions in making digital transactions or trading successful and earning part of revenue share as an incentive. On the other hand, seller gets benefitted by an exponential increase in the revenue.

\subsection{Supply Chain}

Third stage focuses on the creation of dynamic distribution network between the seller and the buyer. The previous stage limited the trading capability of the seller only with his/her trusters similar to the localized re-sell approach, as discussed in the Social Digital Marketplaces and Classifieds Digital Marketplaces. Building upon the previous stage where direct connections were made genuinely interested in the seller, its catalogue and its sales, this stage extends the trading capability of the seller with the stranger beyond geographical boundaries. In case of high value transactions, these people involved in the digital transactions could also conduct in-person inspection in their geographical vicinity for an incentive.

We wish to call the direct connections of the seller in this stage as First-Degree Introducers (FDI). Similar to the seller having direct connections, each participant in our proposed solution will have their own direct connections working on the same principle as discussed in the previous section. FDI's, however, do not broadcast the catalogue of the seller they have partnered with among their direct connections. Instead, they wait for a request from their trusters for introducing them to their preferred vendor for buying a product. This not only ensures that the network avoids unnecessary promotional messages, but also prevents breaking down the resistance of other people through brute force. FDI's are in fact influencers but focus on need-based selling, that is fulfilling the customer's needs on request. Impulsive purchases by their trusters may lead them to debt.

Our Web of Trust* approach allows us to build chain of influencers in a digital transaction. But the number of people involved is based on introduction approach of the social media Friend recommendations (you may also know this person) is a popular feature to add missed connections on the social media. This well researched topic works on the principle that the digital population around the world could be connected by each other via a number of intermediaries or introducers. Facebook Research Team, for example, suggests that each person in the world is connected to every other person by an average of three and a half other people [20]. This is assumed by us to be also true for Open Digital Marketplaces.

We assume that same degree of influencers and number of people involved in a transaction can help develop trustworthy supply chain in our proposed solution. Implying FDI, Second-Degree Introducers, Third-Degree Introducers and so on will be involved in making a digital transaction successful. These introducers or influencers may be spread across 
geographical location but are personally connected to each other. Since influencers are trustees and direct connections are their trusters, buyer is directly trusting the influencer.

Trust of buyer is only limited to his/her influencer. It is never extended to the influencer of the influencer. Therefore, trust in dynamic supply chain which connects strangers (i.e., buyers and sellers) remains direct and localized. Two-way supply chain formed between the buyer and the seller, as discussed in Section 4.3, provides assurance and develops trust among buyer and the seller that their digital transaction is secure.

\subsection{Contract \& Delivery}

Previous stage discusses the formation of supply chain so that buyer and the seller may conduct the transaction with the support of influencers who are rewarded for their contributions. It is envisaged that each influencer will have personal strength or expertise which the buyer would like to leverage, e.g., special taste for food, fashion or goods. However, buyer may have multiple influencers sharing the same interests. From this perspective, the previous stage may propose multiple supply chains to the buyer. In such a case, buyer may select the influencer, that is for example, proposing lowest price, few influencers in the chain, personal inclination towards one or the need for geographical vicinity in case of desired in-person inspection.

Once the buyer has selected an influencer to go ahead with the transaction and decided on the supply chain, e-contract is signed with all the parties involved. Written agreement further develops confidence in the digital transaction. Such a contract for example, will record activities carried out by the influencers, buyer and seller obligations, clauses associated with activity executions, handling of exceptions when there is a clause violation, guarantees, payments and commitments, in-person inspection, obligations and jurisdiction to enable successful transaction incorporating democracy and trust requirements. Any automated system to manage workflows could be used to co-ordinate and execute e-contract. This is digitally signed by all the parties involved, influencers, buyer and the seller.

With contract in place, similar to underwriting in the real world, buyer seeks a bank guarantee (or from financial institution) as a further affirmation of trust in the transaction. It also further mitigates reliance on the marketplace owner and diminishes its authority over the digital transactions. Buyer may transfer an agreed amount to the bank who may hold this amount until the agreement is honored. All the parties are notified of the confirmation of guarantees and seller fulfils the need (e.g., by dispatching the product).

\subsection{Rewards and Dispute}

Buyer, on receiving the product, provides a fulfillment update which is notified to all the parties involved in the transaction so that all could be rewarded as per their contracts. Institution providing guarantee, releases the funds into respective accounts, updates and successfully closes the digital transaction. Repeated successful transaction, between the buyer and the seller may increase their confidence among each other. We envisage that over the time, the role of influencers may diminish. If a buyer is repeatedly satisfied with the seller or vice-versa, one or both may decide to update their trust levels to the direct connections as happening in the real-world [21].

Any party involved in the transaction can raise a dispute. Disputes may arise if any of the parties involved are not satisfied. It may be for example, because that the seller sends a damaged item or buyer falsifies of receiving a damaged item. Automated system will be able to take a fair decision as per the set e-contract. Since the proposed marketplace is democratic in nature, users can, for example, vote to form an independent body consisting of influencers, buyers, seller and government officials to act as an overseer of the proposed Open Digital Marketplace. Members may change from time to time and contribute in resolving escalated disputes (as per the contracts) instead of the current marketplace owners. Complex cases of disputes can be further escalated by the independent body to local jurisdiction and court.

A failed transaction may affect reputation of the influencer, the trust score may decline or the affected party may opt out from the pool of direct connections of the trustee. Thus, affecting the income of influencer. However, if the dispute is resolved, especially with the interference of the influencer, trust will be enhanced and opportunities may broaden for the influencer. Thus, influencers have an incentive to provide guarantees where he/she is confident.

\section{Implementation and Discussion}

Proposed solution was implemented in Python and Django was used for building a mobile optimized web application, srifindia.com. MySQL database used for creation of various tables and storing the data. Our users were not required to install application on their devices.

Debian GNU/Linux server was used to host the service. FastCGI Process Manager were responsible for handling concurrent requests, load management and continuous running for handling further incoming requests. Over a thirty-day test window, on average 178 hits per day contributed to approximately 2260 interactions with the service every day. All the users of the test Digital Marketplace were provided with the feature to manage trustees and discover their trusters.

Three categories of businesses consisting of a FMCG Store, Restaurant and Parlor were selected in the geographic vicinity of $150 \mathrm{kms}$ for the experiment. These represented service-based businesses which required people to visit their physical store to receive the service, product-based business where people could order and receive delivery at their preferred location and a combination of both. All together 90 sellers participated in this experiment.

Multiple databases were used. Registration records were handled with CustomUserManager where users were abstracted into sellers and buyers and their details were stored 
in the Registration Table. Second table recorded sellers' catalogue, product/service details, description and prices. Third and Fourth table recorded trustees and trusters along with their preferred type of transaction such as food. Supply Chain table recorded the chain of influencers for a given transaction including buyer and seller. Contracts table stored the e-contract along with the agreed compensations for all the parties involved in a digital transaction. Disputes table recorded all the disputes along with the record of resolutions.

Need based buyers transacted in the following manner. Let us suppose that a person has a need for a pack of breakfast bars. A message will be sent to the trustees preferred for FMCG. Let us assume that only one of the trustees is in a position to recommend the seller, he/she sends response with the quote, seller and his/her reward ("fee"). If the buyer chooses to accept the supply chain, e-contract is digitally signed by all the parties involved. On the other hand, if multiple trustees recommend multiple (different) sellers, buyer may manually or automatically choose the supply chain from the returned list of trustees based on one or more criteria such as lowest price, no previous disputes, number of intermediaries involved, etc.

Once the contract is finalized, payment is made by the buyer to the digital wallet (used only for experimentation purposes and is suggested to be replaced by a financial institution who will underwrite the digital transaction). Seller is notified to deliver pack of breakfast bar to the desired location of the buyer. On receiving the goods, buyer updates the transaction status. At this moment, all the parties are compensated as per the agreed contract. Influencers were rewarded with redeemable discount and food vouchers for this experiment.

However, if buyer or seller is not satisfied and raises the dispute, then an automated algorithm verifies the violations of the agreement and acts accordingly. Complex disputes are sent to be studied by a person (to be replaced by an independent body or in the court of law, if further referred) for a verdict and refunds. Across the geographical boundaries, influencer may be requested to conduct in-person inspections for resolving the dispute or even to mitigate the risk by conducting a visit prior to signing the contract. But such a situation did not arise during our experiment.

Each successful transaction added one trust point to all the parties involved. Unsuccessful transaction deducted one point from the parties that breached the agreement, including the influencers for recommendations leading to failure. Raise of disputes (e.g., delivery of wrong item from FMCG store) were flagged to users for notifying caution in selecting the supply chain during transaction. Whilst the disputes are resolved, users were allowed to execute other transactions as happening in the real world.

Sellers used WhatsApp to advertise their catalogue in order to inform, persuade and remind their own customers ("trusters") about their products and services. Offers were sent every day over the thirty-day test window by the sellers to their trusters who opted in by accepting the terms and conditions of the experiment. Many of the service-based sellers reported an increase in footfalls in their physical stores.
Small retailers reported ease of doing business as there was no accompanied listing fee, neither any promotional fee and nor the autonomy of the Digital Marketplace. Sharing the revenue with their trusters helped them increase their revenue and customers.

\section{Conclusion}

There has been a growing concern on the supremacy and steamrolling by the Digital Marketplaces. Undemocratic environment, where buyers and seller does not have much voice to say but rather accept the decisions of the digital market owner is leading to an insecure digital environment. Many small businesses are not able to compete with the marketplace owners and are either losing the business or are closing down. In order to overcome this, a new solution for Open Digital Marketplaces was proposed in this paper that models the real world.

Marketplace owner does not exercise any autonomy in the proposed solution. Instead, that role is transferred to users of the marketplace. Sellers leverage their trusters (e.g., existing customers in the real-world), re-ignite their interest and share revenue with them to recommend their products and services within their social circles, irrespective of the size of their social circles. This not only creates new opportunity in the emerging gig economy, introduce fair competition in the market but also gives back control to the users of the marketplace in a very democratic manner.

By modelling real world connections, the digital transactions are made trustworthy, direct and localized. Trust in the marketplace owner is shifted to trusting the influencer who is recommending the seller and replaces the assumption that the user is purchasing the product from the marketplace. Influencers help overcome digital transactions across geographical boundaries and even conduct in-person inspections. Over the time, the trustee-truster relationship or buyer-seller relationship may strengthen which otherwise is currently being marginalized by the current marketplace owners.

As the role of marketplace owners is diminished with our proposed solution, we envisage that the governments will take this opportunity to regain their control and regulate such open markets as in the real world. They may decide and work together with support of an independent global body to enable their policies and trade across geographies. Participation of financial bodies, especially in underwriting digital transactions and sentiments of users based on experience, may open new opportunities for investors in Micro, Small \& Medium Enterprises. It may also help in transforming unorganized sector into an organized sector.

This experiment has now been closed, our team is working on a new version but type of sellers, their service and product-based catalogues could be viewed on SrifIndia Digital Market. As the objective of World Wide Web information system was to find, share and make documents accessible across people, our on-going research is focusing on identifying sellers and their catalogues over network layer to 
create a dedicated Worldwide Open Digital Marketplace for finding, selling and purchasing service or products. As Artificial Intelligence and Artificial Internet of Things is becoming a reality, such a solution will further create opportunities for people and society in general.

\section{References}

[1] T. Berners-Lee, "30 years on, what's next \#For The Web?", World Wide Web Foundation, 2019.

[2] J. Betzing, D. Beverungen and J. Becker, "Design Principles for Co-Creating Digital Customer Experience in High Street Retail", Proceedings of Multikonferenz Wirtschaftsinformatik, 2018.

[3] N. Islam, "Making Digital Marketplaces Fairer", SSRN, 2018.

[4] M. Ciocca, "Online Product Localization: Challenges and Solutions in Global Online Marketplaces", Digital and Social Media Marketing, pp. 275-282, 2019.

[5] I. Hong, "Predicting Consumer Trust in an Intermediary in B2C Online Marketplaces: Insights from the Korean Experience.", in Novel Theories and Applications of Global Information Resource Management, IGI Global, 2020, pp. 154-183.

[6] M. Goyens, "Effective Consumer Protection Frameworks in a Global and Digital World", Journal of Consumer Policy, 2019.

[7] H. Subramanian, "Decentralized blockchain-based electronic marketplaces", Communications of the ACM, vol. 61, no. 1, pp. 78-84, 2017.

[8] B. Edelman and A. Stemler, "From the Digital to the Physical: Federal Limitations on Regulating Online Marketplaces", Harv. J. on Legis, vol. 56, p. 141, 2019.

[9] K. Kapanova and K. Koidl, "Towards a model of interpersonal trust in Social Media Applications", in Proceedings of the 5th EAI International Conference on Smart Objects and Technologies for Social Good, Valencia, Spain, 2019.
[10] D. Holtz, D. MacLean and S. Aral, "Social Structure and Trust in Massive Digital Markets", in International Conference on Information Systems (ICIS). Association for Information System, 2017.

[11] Y. Asim, A. Malik, B. Raza and A. Shahid, "A trust model for analysis of trust, influence and their relationship in social network communities", Telematics and Informatics, vol. 36, pp. 94-116, 2019.

[12] X. Fan, L. Liu, R. Zhang, Q. Jing and J. Bi, "Decentralized Trust Management: Risk Analysis and Trust Aggregation", ACM Computing Surveys, 2019.

[13] J. Sänger and G. Pernul, "Interactive Reputation Systems", Business \& Information Systems Engineering, vol. 60, no. 4, pp. 273-287, 2018.

[14] A. Fradkin, E. Grewal and D. Holtz, "Reciprocity in Two-sided Reputation Systems: Evidence from an Experiment on Airbnb", Working Paper, 2019. https://andreyfradkin.com/

[15] E. Spitko, "Reputation Systems Bias in the Platform Workplace", BYU Law Review, 2019.

[16] J. Kas, R. Corten and A. Rijt, The role of reputation systems in digital discrimination. The Netherlands: Utrecht University, 2019.

[17] S. Clarke, "Trust*: Extending the Reach of Trust in Distributed Systems", PhD thesis, University of Hertfordshire, 2009.

[18] S. Herald, S. Clarke and B. Christianson, "A non-transitive trust model for key distribution", Journal of Information Assurance and Security, vol. 5, no. 6, pp. 618-625, 2010.

[19] K. Smith, "53 Incredible Facebook Statistics and Facts", Brandwatch, 2019.

[20] S. Bhagat, M. Burke, C. Diuk, I. Filiz and S. Edunov "Three and a half degrees of separation", Facebook Research, 2019.

[21] A. Rezvanian, B. Moradabadi, M. Ghavipour, M. Daliri Khomami and M. Meybodi, "Social Trust Management", Studies in Computational Intelligence, pp. 241-279, 2019. 